\title{
Variables Socioculturales y Cognitivas en el Desarrollo de la Comprensión de Lectura en Lima, Perú
}

\section{Silvia Morales}

Universidad Nacional Agraria La Molina

Silvia Morales es doctora en psicopedagogía por la Universidad Radboud de Nimega, Holanda. Especialista en Educación del Talentoso (Holanda) y Psicóloga Educacional por la Pontificia Universidad Católica del Perú (PUCP). Es miembro del Proyecto "Innovación Educativa" del convenio del Consejo de Universidades Flamencas de Bélgica (VLIR) y la UNALM, en donde se desempeña como investigadora y capacitadora. Es terapista en problemas de aprendizaje. Tiene experiencia como docente universitaria. Investiga la enseñanza en la universidad y la comprensión de lectura; y ha investigado creatividad y producción de textos.

La autora agradece profundamente al convenio para el programa de doctorado entre la Pontificia Universidad Católica del Perú y la Universidad Católica de Nimega (Holanda), especialmente a los doctores Ludo Verhoeven y Jan van Leeuwe; así como al Dr. Santiago Cueto de la PUCP, quienes brindaron permanente asesoría y apoyo teórico, técnico y financiero para llevar a cabo esta investigación. 


\title{
Variables Socioculturales y Cognitivas en el Desarrollo de la Comprensión de Lectura en Lima, Perú
}

\begin{abstract}
Resumen
Esta investigación está compuesta por tres estudios, en los cuales participaron estudiantes y profesores de dos niveles socioeconómicos de Lima. La comprensión de lectura se midió mediante una adaptación de la prueba PIRLS Reading Literacy test. El Modelo de Ecuación Estructural y el ANOVA se utilizaron para analizar los datos. El Estudio 1 examinó en qué medida la variación en comprensión de lectura en niños de cuarto grado puede ser predicha por su nivel en motivación hacia la lectura, estrategia, vocabulario y decodificación; y por variables antecedentes: madurez intelectual, género, clima alfabético del hogar y nivel socioeconómico. El Estudio 2 investigó en qué medida el desempeño de los estudiantes en comprensión de lectura, decodificación, vocabulario y motivación hacia la lectura al final del quinto grado se puede predecir por las mismas variables al inicio del año y por las variables antecedentes. El Estudio 3 examinó si el desarrollo de la comprensión de lectura y de la motivación hacia la lectura de 202 niños de quinto grado puede mejorar mediante un programa que fomenta el compromiso por la lectura. Los resultados de los tres estudios mostraron que las variables que contribuyen con mayor fuerza al desarrollo de la comprensión de lectura son vocabulario, decodificación, motivación hacia la lectura y nivel socioeconómico, el cual está ligado al clima alfabético del hogar. Y, en un segundo nivel, contribuyen el género y la madurez intelectual, que sería más relevante en niños mayores. Estos resultados resaltan la necesidad de una perspectiva motivacional y sociocultural al diseñar programas de intervención en lectura.
\end{abstract}

Palabras clave: lectura, vocabulario, motivación, condición social, formación docentes primaria 


\title{
Socio-Cultural and Cognitive Variables and Reading Literacy Acquisition in Lima, Peru
}

\begin{abstract}
This research is composed of three studies where students and teachers from two social economic groups in Lima participated. Reading literacy was measured using an adaptation of the PIRLS Reading Literacy test. Structural Equation Modelling and ANOVA were used to analyze data. Study 1 examined to what extent the variation in reading literacy in fourth grade children can be predicted from their reading motivation, strategy, vocabulary, and word decoding, and from background variables: intellectual maturity, gender, home literacy climate, and social economic status. Study 2 investigated to what extent the performance of students on reading literacy, word decoding, vocabulary and reading motivation at the end of the fifth grade can be explained from the same group of variables measured at the beginning of the fifth grade and from the background variables. Study 3 examined if the development of reading literacy and reading motivation of 202 fifth graders can be improved by means of an engaged reading programme. Results of all three studies showed that the strongest variables that contribute to reading literacy development in students are vocabulary, word decoding, reading motivation and social economic status which is related with home literacy climate. A second group of contributors to reading literacy development consists of gender and intellectual maturity, which might be important more in older than in younger children. The results evidences the importance of an engagement perspective and a cultural diversity approach while designing intervention programmes on reading literacy development.
\end{abstract}

Keywords: reading, vocabularies, motivation, social status, primary teacher education 
- Cuáles son los factores que contribuyen al desarrollo de la comprensión de lectura en estudiantes de Lima?

Esta pregunta motivó la presente investigación llevada a cabo en Lima, una ciudad que presenta un panorama complejo en los ámbitos sociales y educativos. En este contexto, el desarrollo de la comprensión de lectura es un tema que ha tomado alta relevancia en los últimos años y se encuentra actualmente entre los temas urgentes del Ministerio de Educación del Perú (MED) (MED, 2000, 2007).

\section{Panorama Educativo del Perú}

En el Perú, aproximadamente el 10,9\% de la población mayor de 15 años es analfabeta (MED, 2007). Esta cifra representa el 16,6\% de la población femenina y $5,9 \%$ de la población masculina; así como $5,6 \%$ de la zona urbana y $24 \%$ de la zona rural. Cabe resaltar que, desde la década de los ochenta, se ha logrado un gran progreso en el Perú en relación con el acceso (la matrícula) en la educación primaria. La cobertura de la población de los niños de 6 a 11 años en el sistema educativo ha alcanzado cifras cercanas a la universalización (96,5\% en 2006), sin presentar grandes brechas por género, área o nivel de pobreza. A pesar de este avance, la gran preocupación actual es que la mayoría de niños peruanos no alcanza el nivel mínimo en comprensión de lectura de acuerdo con lo que exige el currículo educativo nacional. Los resultados de la Evaluación Nacional (EN) 2004 muestran problemas importantes de calidad y de equidad en los logros de los estudiantes en comprensión de lectura y matemática en todos los grados evaluados. Esto significa que la mayoría de estudiantes no alcanza los niveles de desempeño esperados para el grado. Más aun, los niños de grupos socioeconómicos bajos y los de origen indígena suelen obtener puntajes más bajos que los estudiantes provenientes de niveles socioeconómicos más altos y de familias que hablan el español, en contraste con las que hablan quechua (Benavides, 2007). Como lo han manifestado algunos autores (MED, 2007), estos resultados también llevan a reflexionar sobre la necesidad de que el sistema educativo peruano determine los estándares de desempeño del sistema en el ámbito nacional.

Otro factor importante para tomar en cuenta es la deserción en la educación primaria, que afecta de manera negativa la adquisición de las habilidades básicas en lectura (así como en escritura y aritmética). Se necesita un mínimo de cuatro años de escolarización continuos para alcanzar ese nivel, el 
cual es fundamental para que el alumno continúe con su formación. Estudios realizados (MED, 2007) han señalado que solo el $44,8 \%$ de los niños inscritos en educación primaria logrará cursar los cuatro primeros años de escuela de manera constante, los cuales se consideran indispensables para adquirir capacidades duraderas en materia de lectura, así como de escritura y aritmética.

Por otro lado, es importante agregar que los estudiantes peruanos han obtenido un rendimiento más bajo que los estudiantes de un gran grupo de países latinoamericanos, como lo han evidenciado las últimas evaluaciones internacionales sobre temas escolares; específicamente, la evaluación del Laboratorio Latinoamericano de Evaluación de la Calidad Escolar (LLECE) (ver Unidad de Medición de la Calidad, boletín web) y la evaluación del Programme for International Student Assessment (PISA) (OECD y UNESCO, 2003).

Frente a esta realidad, el Ministerio de Educación (2007) se ha propuesto como objetivo fundamental contribuir a mejorar los niveles de logro de los niños de educación primaria, promoviendo una formación integral que desarrolle en los niños valores, actitudes, habilidades sociales y habilidades básicas de resolución de problemas, lectura, escritura y operaciones aritméticas.

Por otro lado, se ha evidenciado baja calidad en los programas de formación docente. Dentro del plan para mejorar la educación en el Perú, el Ministerio de Educación ha incluido el control de la calidad de los centros de formación para docentes (MED, 2000) y, por otro lado, revalorar la carrera pedagógica y elevar los salarios (MED, 2004). Durante los últimos años se han dado esfuerzos para cumplir esas metas, como fue la creación del colegio profesional de profesores, cuya primera junta directiva inició sus actividades en 2006 (Colegio de Profesores del Perú, boletín web).

\section{El Factor Sociocultural y la Educación en el Perú}

Debido a la diversidad cultural del Perú y, específicamente, de Lima, esta investigación presenta una aproximación sociocultural. En Lima se han identificado cinco niveles socioeconómicos (Apoyo, Opinión y Mercado, 2003); en este estudio hemos considerado dos de ellos.

A este respecto, diversos estudios han señalado que una de las dificultades al diseñar programas de enseñanza es la gran diversidad entre los estudiantes de diferentes lugares. Varios estudios previos (p.e., Heath, 1982, 1983; Serpell, 2001; Street, 2001) han encontrado que la diversidad sociocultural ha tenido un gran impacto en el desarrollo de habilidades de lectura. Street 
(2001) sostuvo que la alfabetización (literacy) es una práctica social que varía de un contexto a otro y es parte de la conducta y conocimiento culturales, no simplemente una competencia técnica que se adhiere a las personas. Se relaciona con la identidad cultural de las personas, su sentido del self, su perspectiva del conocimiento y del mundo, su epistemología. En el Perú sucede que muchos niños que tienen la misma edad no presentan el mismo nivel de desarrollo en el lenguaje (p.e., Cueto, Jacoby y Pollitt, 1997a; Ramírez, 1997). Los hallazgos de Ramírez muestran grandes diferencias entre los niños de las zonas rurales y urbanas al inicio del primer grado. Asimismo, en el área urbana se han encontrado diferencias entre los niños de niveles socioeconómicos bajo y alto (p.e., Carreño, 2000; Morales, 1999). Estos resultados sustentarían la noción del lenguaje como un sistema social.

Con el propósito de entender las diferencias presentadas, es importante conocer que, en las áreas urbanas, especialmente en hogares que pertenecen a los niveles socioeconómicos medios y altos, los niños preescolares experimentan el lenguaje de una manera similar a la manera en que lo experimentarán más adelante en el colegio. Ellos escuchan cuentos, se familiarizan con el vocabulario, los números, los símbolos. Antes de aprender a leer o escribir, ellos van entendiendo el significado de la alfabetización. De manera contraria, en las áreas rurales del Perú, los niños no tienen contacto con material escrito, porque no hay ningún material impreso en casa, no hay avisos ni señalizaciones en las calles. Ellos están más familiarizados con la naturaleza y practican la tradición oral; y cuando ingresan al colegio, la actividad de alfabetización es totalmente nueva para ellos (Zavala, 2001).

Por otro lado, en el caso de las zonas urbanas, los estudiantes de los colegios privados permanecen, en promedio, más tiempo en el colegio que sus pares de colegios estatales: más horas por día y más días durante el año escolar. Aproximadamente, entre $30 \%$ y $50 \%$ más tiempo. Estos hallazgos cobran importancia, pues se ha encontrado una correlación entre desempeño escolar en escuelas rurales y el tiempo que se pasa en el colegio (Cueto, Jacoby y Pollitt, 1997b). Esto se complementa con el estudio de Walberg y Wang (1989), quienes han sostenido que la cantidad de instrucción constituye un elemento necesario; sin embargo, es insuficiente por sí sola para producir el aprendizaje.

Otro de los elementos relacionados con la diversidad sociocultural que contribuyen a la adquisición de la lectura en el Perú es el género. La falta de oportunidades educativas en algunas zonas del país y factores culturales y familiares contribuyen a la proporción mayor de analfabetismo en mujeres (Anderson, 1993; MED, 2004). Dentro de la familia, los factores más importantes 
son la falta de recursos económicos, la necesidad del trabajo en la casa para las niñas, la resistencia de los padres hacia los colegios mixtos, enfermedad, matrimonio a edades tempranas y el nivel educativo de los padres (van der Western, 1994).

\section{Motivación y Lectura}

Adicionalmente, otro elemento importante en esta investigación fue la perspectiva motivacional. La motivación ha sido considerada como un factor determinante al explicar el proceso de lectura y la adquisición de las destrezas de lectura (Verhoeven y Snow, 2001; Wigfield y Guthrie, 1997). En los estudios de Wigfield (1997), la motivación hacia la lectura ha sido definida como multidimensional, y se relaciona tanto con la frecuencia de lectura como con la calidad del desempeño en sí.

Uno de los conceptos importantes que han surgido en torno a la lectura es el compromiso/implicación (engagement) por la lectura. En 1995, Cambourne (Guthrie y Wigfield, 2000) sostuvo que el compromiso implicaba mantener un propósito, buscar comprender, creer en la propia capacidad y asumir la responsabilidad por el aprendizaje. La mayoría de los investigadores coincide en que los lectores se encuentran constantemente tomando decisiones y sus afectos, lenguaje y cognición contribuyen en su desempeño lector. Entonces, las personas leen un texto no solamente porque están en capacidad de hacerlo sino, también, porque están motivadas para hacerlo. En este sentido, leer de manera comprometida (engaged reading) es una actividad estratégica y conceptual, así como motivadora e intencional. Desde una perspectiva cognitiva, los lectores efectivos se encuentran tomando decisiones y escogiendo estrategias deliberadamente para comprender un texto.

Asimismo, para la construcción del significado, la interacción con pares cumple un rol importante. Desde esta aproximación, el compromiso se da cuando se activan tres dimensiones: la cognitiva, la social y la motivacional. Tal como han señalado Guthrie y Wigfield (2000), los lectores comprometidos coordinan sus estrategias y conocimiento (cognición) dentro de una comunidad literaria (el ámbito social) con el fin de cumplir con sus metas personales y propósitos (el ámbito de la motivación).

Otra de las dimensiones ligadas a la motivación es la autoeficacia. La autoeficacia se refiere a la creencia o certeza de que uno es capaz de realizar una tarea. Los lectores confían en sí mismos y piensan que son capaces de 
desempeñarse en una tarea de lectura de manera eficaz (Wigfield y Guthrie, 1997). Estos pensamientos conducen a los lectores a ser perseverantes mientras lidian con las dificultades de la tarea. Ellos estarán más dispuestos a embarcarse en las estrategias trabajosas que quienes tienen dudas sobre su capacidad (Alexander, Graham y Harris, citados en Garner, 1998).

El valor asignado a la lectura es otro concepto estudiado por Wigfield (1997). Se refiere a la creencia individual en cuanto a que la lectura es una actividad de importancia central para el individuo. Alexander, Graham y Harris (Garner, 1998) han argumentado que los estudiantes estarán más dispuestos a realizar una tarea difícil si ellos creen que esa tarea tiene un valor. Las oportunidades relacionadas con la alfabetización - que incluye la lectura, escritura y el lenguaje en general-que los padres brindan en casa reflejan el valor que ellos asignan a la alfabetización. Por ejemplo, como han afirmado Guthrie y Greaney (1991), los padres que inscriben a su hijo en una biblioteca demuestran que valoran la alfabetización.

Por otro lado, también se han estudiado los efectos de la edad y del género en el desarrollo de la motivación por la lectura. Se ha encontrado que diferentes dimensiones de la motivación disminuyen a lo largo de los años escolares; por ejemplo, el interés general por el colegio, la motivación intrínseca, el impulso continuo por aprender y las expectativas de éxito en diferentes cursos (Wigfield, 1997). Con respecto a estos hallazgos, McKenna, Kear y Ellsworth (1995) encontraron que las actitudes hacia la lectura académica o recreativa se volvían más negativas a lo largo de los años escolares de la primaria. Los alumnos empezaban en el primer grado con un nivel positivo y, en sexto grado, el nivel era de una indiferencia relativa. Asimismo, en el estudio de Wigfield y Guthrie, los alumnos de cuarto grado obtuvieron puntajes mayores que los de quinto grado en motivación hacia la lectura (Wigfield, 1997).

En relación con el género, algunos estudios han evidenciado que las niñas presentan mayor nivel en motivación hacia lectura que los niños. En su estudio, Wigfield y Guthrie (1997) encontraron que las niñas de cuarto y de quinto grado presentaban mayor grado de motivación hacia la lectura que los niños.

\section{Programas Educativos sobre Comprensión de Lectura}

En los últimos años se han desarrollado algunos programas de intervención en comprensión de lectura que han presentado relativamente buenos resultados en grupos de alumnos específicos. A continuación se presentan algunos de ellos. 


\section{Enseñanza Recíproca}

Este paquete instructivo de estrategias múltiples fue diseñado por Palincsar y Brown en 1984 (Brown, Pressley, van Meter y Schuder, 1996). Los estudiantes aplican cuatro estrategias mientras leen: elaborar predicciones, formular preguntas, esclarecer dudas y resumir contenidos. Los estudiantes usan esas estrategias mientras leen en grupos y el profesor va, poco a poco, trasladando la responsabilidad del trabajo al grupo. Los principios de este método se encuentran en la línea de la teoría de Vygotsky relacionada con el aprendizaje socialmente mediado. Así, el método resalta que el trabajo en grupo desde cada uno de los miembros fomentará la internalización de esos procesos. Esta propuesta ha mostrado resultados valiosos en el dominio de las estrategias focales y mejoras medianas relacionadas con una prueba estandarizada de lectura.

\section{Instrucción de Estrategias Transaccional}

Pressley, Goodchild, Fleet, Zajchowsky y Evans (1989, citados en Brown, Pressley, van Meter y Schuder, 1996) estudiaron diversos programas que fomentaban la comprensión de lectura, y concluyeron que una instrucción exitosa de estrategias en el aula involucraba más de lo que se conseguía con los procedimientos controlados de los experimentos. Asimismo, Brown, Pressley, van Meter y Schuder (1996) sustentaron que la comprensión efectiva de nivel básico era transaccional (transactional) en tres sentidos (definidos por Pressley, El-Dinary, Gaskins, Schuder, Bergman, Almasi y Brown en 1992): a) se estimula a los estudiantes a construir significados, usando estrategias que permitan hacer el nexo entre la lectura del momento y el conocimiento previo; b) mucha parte del aprendizaje surge del trabajo en grupos. Los integrantes del grupo construyen el significado trabajando juntos. Esto es aparte del entendimiento individual que adquiere cada alumno; c) los desempeños de todos los miembros del grupo se determinan en parte por los otros miembros del grupo.

Dentro de esta perspectiva, los alumnos construyen el significado al aprender de lectores expertos. El profesor y los alumnos modelan el uso de estrategias entre sí. Por ejemplo, ellos usan el pensar en voz alta al leer. Asimismo, se discute en grupo en qué momento se tiene que aplicar alguna estrategia. El estudio de Brown, Pressley, van Meter y Schuder (1996), en el cual participaron alumnos de bajo rendimiento de segundo grado por un año, mostró resultados positivos 
de la instrucción de estrategias transaccional. Los alumnos construyeron un significado más rico de las lecturas y mostraron mayor conciencia del uso de estrategias y su uso más frecuente en comparación con sus pares. Asimismo, obtuvieron mejores resultados en las pruebas estandarizadas de comprensión de lectura. Es importante notar que los investigadores recalcan que la formación de alumnos que se autorregulen en su aprendizaje llevaría años de experiencias de alfabetización y la metodología de estrategias transaccional sería un buen inicio.

\section{Instrucción de Lectura Orientada a un Concepto (CORI)}

La metodología de CORI (por sus siglas en inglés) fue desarrollada por Guthrie et al. (1996). A continuación, se presentan los principios básicos (Guthrie y Alao, 1997):

Tema conceptual. La instrucción se organiza en torno a un tema interdisciplinario, un concepto, frente al cual varias disciplinas, tales como lenguaje, arte y ciencias, puedan enseñarse de manera simultánea. En este sentido, con el propósito de entender el concepto, se llevan a cabo un gran número de actividades mientras que se enseñan las estrategias cognitivas. El tema conceptual le brinda al estudiante el propósito para leer, investigar y es su guía para alcanzar el dominio. Asimismo, esas metas incrementan el placer y motivación por trabajar el texto y desarrollan el sentimiento de autoeficacia.

Colaboración social. Las interacciones sociales contribuyen al aprendizaje de conceptos. Se presentan actividades en diferentes grupos: individuales, en parejas, grupos pequeños o con toda la clase. Los estudiantes aprenden a coordinar sus esfuerzos, compartir información, ayudar a otros y aprender de los otros. Los ambientes de aprendizaje colaborativos incrementan la motivación.

Interacciones con el mundo real. A través del contacto con objetos concretos o eventos se adquiere de mejor manera un concepto. Al inicio se da el entusiasmo de los alumnos, lo cual incrementa el interés. Luego, estas experiencias contribuyen al entendimiento profundo del tema y a la formulación de mejores preguntas de investigación.

Transferencia de conocimiento. Se incentiva la transferencia de lo aprendido al brindar múltiples ejemplos. Como sostuvieron Anderson, Reder y Simon (citados en Guthrie y Alao, 1997), el aprendizaje está conectado con el contexto en el que se da; por ello es indispensable diseñar procedimientos instructivos que lleven a la transferencia. Se brindan a los alumnos los medios y materiales 
para que se informen, escriban y participen en debates sobre eventos variados.

Autodirección. Se estimula la autonomía de los estudiantes, quienes asumen la responsabilidad por aprender. Ellos tienen la oportunidad de participar en las decisiones y de identificarse con las metas de aprendizaje. Se les deben brindar opciones que sean significativas. Ellos aprenden a elegir el tema, los textos y los medios para aprender.

Autoexpresión. Los estudiantes tienen que articular lo que han aprendido. Se ven a sí mismos como creadores de conocimiento y podrán valorar las estrategias para su aprendizaje (Belenky, Clinchy, Goldberger y Tarule, 1986, citados en Guthrie y Alao, 1997).

Textos interesantes. Se pone a disposición de los alumnos una amplia variedad de recursos de información. Estos materiales tienen que ser coherentes con el nivel cognitivo de los alumnos y ser interesante.

Estrategias cognitivas. La instrucción incluye apoyo para el aprendizaje de las estrategias. Para esto se utiliza el modelamiento, explicación, coaching, discusiones en grupo, práctica guiada y reflexión individual. Las estrategias que se han investigado más son planificación, predicción, inferencia, uso del conocimiento previo, organización, elaboración de síntesis, uso de analogías, elaboración conceptual, pensamiento de causa-efecto en el texto, autoexplicaciones al leer y establecimiento de metas.

Coherencia. La integración se forja mediante el tema conceptual. Así, la instrucción vincula actividades, estrategias para aprender, materiales, colaboración social y presenta los entendimientos alrededor del tema. Cuando el currículo ofrece integración y pertinencia, los estudiantes tendrán mayor facilidad para integrar sus aprendizajes en esquemas de significado (Beane, 1995, citado en Guthrie y Alao, 1997).

Las investigaciones realizadas con el programa CORI en diez salones de clase de los grados tercero y cuarto usando grupo de control, mostraron que los alumnos que participaron en el programa obtuvieron puntajes más altos en motivación hacia la lectura que sus pares del grupo control. Asimismo, estos niños se mostraron más dispuestos a incrementar la cantidad de material de lectura y ampliar los temas; y también usaron más estrategias para reducir las distracciones mientras leen y mayor interés por compartir libros con sus amigos. Por otro lado, estos niños obtuvieron puntajes más altos en pruebas estatales de lectura, escritura, lenguaje, ciencias y ciencias sociales. 


\section{Objetivos de la Investigación}

Con la presente investigación se quería llegar más allá de los resultados de estudios previos realizados en Lima (p.e., Arakaki, 2004; Carreño, 2000). Asimismo, se pretendía recabar información que fuera posible comparar con los resultados de otros contextos. Por primera vez, un grupo de variables cognitivas y de contexto relacionadas con la comprensión de lectura fueron evaluadas juntas en un modelo; asimismo, se consideró una aproximación de desarrollo, así como un programa de intervención; entonces, no solo los estudiantes fueron investigados, también participaron los docentes.

La investigación consistió en tres estudios:

El Estudio 1 consistió en un diseño trasversal que examinó en qué medida la variación en comprensión de lectura en 314 alumnos de cuarto grado se podía predecir a partir de su nivel en motivación hacia la lectura, estrategias de lectura, vocabulario y decodificación, por un lado, y por su madurez intelectual, género, clima alfabético del hogar y el estatus socioeconómico, por otro lado.

A partir de la primera aproximación a la muestra y a las variables, el Estudio 2 investigó la variación sociocultural en el desarrollo de la lectura de 331 alumnos de quinto grado de dos grupos socioeconómicos en Lima. Se investigó en qué medida los resultados de los niños en la comprensión de lectura, decodificación, vocabulario y motivación hacia la lectura al final del quinto grado se pueden explicar por el mismo grupo de variables al inicio del quinto grado $y$, adicionalmente, por las variables de fondo, a saber, madurez intelectual, género, clima alfabético del hogar y estatus socioeconómico.

El Estudio 3 completó la investigación con una perspectiva instructiva, examinando si el nivel de la comprensión de lectura y de la motivación hacia la lectura de 202 alumnos de quinto grado de Lima pertenecientes a dos grupos socioeconómicos pueden ser mejorados a través de un programa de intervención de motivación-compromiso hacia la lectura (reading engagement).

\section{Metodología}

\section{Participantes}

Los participantes provinieron de dos grupos socioeconómicos descritos como los niveles C y D por Apoyo, Opinión y Mercado (2003) y que, en el 
presente artículo, serán denominados medio y bajo. Se decidió trabajar con dos grupos socioeconómicos con el propósito de realizar una comparación en ese ámbito, y se eligieron esos dos grupos porque correspondían a los grupos denominados "típicos" - es decir, los más comunes- de Lima. Se eligieron 11 colegios de Lima relacionados con estos dos grupos socioeconómicos y, de cada colegio, se seleccionaron al azar las aulas de los grados que se deseaba examinar: cuarto grado para el Estudio 1 y quinto grado para los Estudios 2 y 3. Asimismo, para el Estudio 3 se consideró un aula paralela de control en el mismo colegio, para así asegurar que sean alumnos comparables.

En el Estudio 1 participaron 314 alumnos de cuarto grado, en el Estudio 2 intervinieron 331 alumnos de quinto grado y, en el 3, se contó con 202 alumnos de quinto grado.

En las Tablas 1 y 2 se presentan las características de la muestra para cada estudio.

Tabla 1

Características de la Muestra para los Estudios 1 y 2

\begin{tabular}{ccc}
\hline \multirow{2}{*}{ Características } & Estudio 1 (Cuarto) & Estudio 2 (Quinto) \\
\cline { 2 - 3 } Edad $\dot{x}$ & $(\mathrm{n}=314)$ & $(\mathrm{n}=331)$ \\
Total NSE medio & 9,4 & 10,6 \\
Niños & 101 & 134 \\
Niñas & 52 & 75 \\
Total NSE bajo & 49 & 59 \\
Niños & 213 & 197 \\
Niñas & 119 & 119 \\
\hline
\end{tabular}

Tabla 2

Características de la Muestra para el Estudio 3

\begin{tabular}{lccc}
\hline \multirow{2}{*}{ Características } & \multicolumn{3}{c}{ Estudio 3 (Quinto) } \\
\cline { 2 - 4 } & Control & Intervención & Total \\
\cline { 2 - 4 } & 66 & 51 & 117 \\
\hline NSE medio & 44 & 41 & 85 \\
NSE bajo & 63 & 46 & 109 \\
Niños & 47 & 46 & 93 \\
Niñas & 4 & & \\
\hline
\end{tabular}




\section{Instrumentos}

- Comprensión de lectura. El test de comprensión de lectura PIRLS ${ }^{1}$ fue utilizado para evaluar comprensión de lectura en los niños. El test evaluó las siguientes habilidades: focalizar y recuperar información explícita, elaborar inferencias sencillas, interpretar e integrar ideas, así como examinar y evaluar el contenido, lenguaje y elementos textuales (International Association for the Evaluation of Educational Achievement, 2000). Las preguntas fueron de dos formatos: elección múltiple y respuesta abierta. Las preguntas de elección múltiple tenían 4 opciones de respuesta, de las cuales solo una era la respuesta correcta. Esta se calificaba con un punto. La respuesta abierta era calificada con uno, dos o tres puntos, dependiendo de la profundidad de esta. Todos los textos e ítems mostraron "muy buenas características psicométricas con un amplio rango de niveles de dificultad e índices de discriminación adecuados" (Martin, Mullis y Kennedy, 2003, p. 10). En el presente estudio se utilizaron dos subtests de la prueba PIRLS: The Hare Heralds the Earthquake (un texto narrativo) y The River Trail (un texto informativo). Estos subtests fueron traducidos del inglés al español; luego, fueron adaptados a los estudiantes de Lima mediante una evaluación piloto. En la evaluación piloto participaron 137 alumnos y se obtuvo un coeficiente de confiabilidad $\alpha$ de ,76 para el subtest narrativo y de ,72 para el subtest informativo. Un test consistía en la historia de una liebre seguida de 11 preguntas sobre esa historia. El otro test consistía en un folleto que daba información sobre un viaje por el campo, seguido de 11 preguntas sobre esa información. En total eran 22 preguntas.

- Estrategias de lectura. Se utilizó una adaptación del instrumento Reading Strategies Questionnaire de van Elsäcker (2002) con el propósito de evaluar el uso de estrategias de lectura en los niños. El cuestionario está conformado por 28 ítems que exploran con qué frecuencia (p.e. siempre, a veces, casi nunca) el estudiante usa determinadas estrategias ya sea antes, durante o después de leer un texto. Durante la prueba, que se tomó de manera grupal, el evaluador leyó a los alumnos cada pregunta en voz alta y después de cada pregunta dejó un periodo para que los niños seleccionaran su respuesta. 
Durante el proceso de adaptación del instrumento se realizó una prueba piloto con 140 niños de cuarto grado para verificar la validez y confiabilidad de las preguntas. Se obtuvo un coeficiente de confiabilidad alfa de ,87. Asimismo, se realizó el análisis factorial, mediante el cual se identificaron dos dimensiones del instrumento: estrategias de monitoreo y estrategias de rutina. El coeficiente de confiabilidad para cada factor fue ,79 y ,67, respectivamente. El factor estrategias de monitoreo se refiere a las estrategias que requieren pensar o autorreflexión y un monitoreo permanente de la comprensión. El factor estrategias de rutina comprende acciones mecánicas que frecuentemente son fomentadas por los profesores (ver Anexo).

- Vocabulario. La prueba Peabody Picture of Vocabulary Test fue diseñada por L. M. Dunn y L. Dunn en 1959 y la versión para Hispanoamérica utilizada en el presente estudio, denominada Test de Vocabulario en Imágenes Peabody fue desarrollada por L. M. Dunn, Padilla, Lugo y L. Dunn en 1986 (Dunn, Padilla, Lugo y Dunn, 1986). Este instrumento mide vocabulario receptivo y se puede aplicar a niños desde la edad de dos y medio hasta los dieciocho años. El material consiste en un cuadernillo de 125 páginas. Cada página evalúa una palabra usando cuatro figuras, donde solo una de ellas representa a la palabra correcta. Durante la evaluación oral individual se le pidió al niño que indicara cuál de las cuatro figuras representaba a la palabra dicha de manera oral.

- Decodificación de palabras. Se utilizó una versión adaptada de la prueba de Decodificación de Un Minuto de Thorne (1991). La prueba medía la precisión y velocidad con que los estudiantes leían una lista de palabras en un tiempo dado de un minuto. La prueba de Thorne fue construida con el fin de evaluar a niños de primer grado de primaria. Se realizó una aplicación piloto a 60 niños de colegios estatales y privados y se calculó el coeficiente de confiabilidad a través del procedimiento test-retest, el cual fue, 96 .

- Motivación hacia la lectura. Con el fin de evaluar la motivación de los niños para leer, se elaboró un cuestionario de 52 preguntas, basado en dos cuestionarios anteriores: Measure of Children's Motivation for Reading, de Wigfield, Guthrie y Mc Gough (1996), y Motivation to Read Profile, de Gambrell, Martin Palmer, Codlin y Anders Mazzoni (1995). Durante la 
evaluación, el evaluador leía cada pregunta a los alumnos en voz alta y daba una pausa para que los alumnos respondieran, eligiendo una de cuatro alternativas. Antes de usar este cuestionario con la muestra, se realizó una evaluación piloto con 137 alumnos. El coeficiente de confiabilidad $\alpha$ que se obtuvo fue de, 90 . El análisis factorial mostró cuatro factores subyacentes: autoeficacia, interacción social, valor asignado a la lectura y leer como entretenimiento. Los coeficientes de confiabilidad para los cuatro factores fueron ,85, ,85, , 81 y ,72, respectivamente. A continuación se describen esos factores.

La autoeficacia se refiere a la percepción de la persona sobre su propio desempeño en lectura. Interacción social se refiere a la medida en que la persona experimenta la lectura como una actividad social. Valor asignado a la lectura se refiere al valor de la lectura en relación con el desarrollo personal y académico. Leer como entretenimiento implica la medida en que los niños consideran la lectura como una actividad placentera.

- Clima alfabético del hogar. El cuestionario sobre el clima alfabético del hogar fue adaptado de la versión original de van Elsäcker (2002). Las preguntas se referían a la cantidad de tiempo que los niños y padres pasan leyendo, la disponibilidad de materiales de lectura y la frecuencia con que se realizan en casa actividades relacionadas con la lectura o alfabetización. El cuestionario original fue traducido del holandés al español y se realizó una aplicación piloto con niños de características similares a las de los participantes de la muestra. De estos resultados se elaboró la versión final del cuestionario. Este consistió en 17 preguntas, las cuales mostraban entre 3 y 6 opciones de respuesta (ver Anexo). El índice de confiabilidad total del cuestionario fue de,72.

- Madurez intelectual. Con el propósito de obtener un índice de la madurez intelectual de los niños se usó el Test de Dibujo de una Persona de Goodenough (versión para Latinoamérica, 1969). Durante la prueba, se le pedía al niño que dibujara una persona. Los criterios de corrección incluyeron 73 criterios para el dibujo de hombre y 71 criterios para el dibujo de una mujer.

- Nivel socioeconómico. La información relacionada con los antecedentes socioeconómicos de los participantes se recogió mediante un cuestionario socioeconómico, el cual fue construido sobre la base de los criterios 
utilizados por Apoyo, Opinión y Mercado (2001, 2003). Los ítems exploraban el nivel de educación y ocupaciones de los miembros de la familia, así como las características de la casa. El cuestionario contenía 18 preguntas que los participantes respondieron en compañía de sus padres o con ayuda de sus maestros.

\section{Procedimiento}

Después de definir el plan de la investigación y sus etapas, se eligieron los instrumentos. Debido a que muchos de los instrumentos no estaban adaptados a los objetivos de la investigación o a la población del Perú o de Lima e, inclusive, algunos de ellos estaban en otro idioma (inglés u holandés), se continuó con el proceso de elaboración y de adaptación de los instrumentos al contexto peruano.

El proceso de adaptación del instrumento implicó la traducción, los ajustes de la redacción y contextualización, así como la evaluación piloto en una muestra similar a la de la muestra objetivo. Después se realizó el análisis cualitativo y estadístico de los ítems de la evaluación piloto para elegir los aquellos más confiables y definir su redacción. También se efectuó el análisis factorial para el instrumento de estrategias de lectura. Por último, se elaboró la versión final del instrumento.

Cuando la muestra estuvo definida, se inició la primera medición con alumnos de cuarto grado (Estudio 1). Los resultados de esta primera fase fueron analizados a través del Cálculo de Correlaciones de Pearson y usando el Modelo de Ecuación Estructural (Amos 4.0). Se realizó un procedimiento paso a paso con el fin de examinar la variación de la variable comprensión de lectura. Parte de este procedimiento incluyó el análisis de regresión. Adicionalmente, se revisaron instrumentos y aspectos de la metodología. Con el propósito de verificar el ajuste del modelo, se utilizaron la prueba de chi cuadrado, el índice de bondad de ajuste, el índice de bondad de ajuste ajustado, el índice de ajuste normado y la raíz cuadrada del error de aproximación. Teniendo en cuenta esos primeros resultados, se continuó el año siguiente con la segunda medición al inicio del año escolar y la tercera medición al final del año escolar con alumnos del quinto grado (Estudio 2). Estos resultados se también se analizaron mediante Correlaciones de Pearson. Al utilizar el Modelo de Ecuación Estructural se verificó el ajuste del modelo y se procedió paso a paso con el propósito de analizar la relación entre la variable 
comprensión de lectura y las otras variables a lo largo de dos momentos de evaluación. Adicionalmente, se examinó la estabilidad de los instrumentos a lo largo del tiempo

Paralelamente, al inicio de esta segunda fase, se realizó la capacitación de los profesores durante una semana en la metodología para mejorar la comprensión de lectura de sus alumnos sobre la base de la perspectiva de la motivación-compromiso hacia la lectura (reading engagement). Los alumnos de quinto grado cuyos profesores participaron en ese entrenamiento, fueron evaluados antes y después de ser expuestos a la metodología que sus profesores desarrollaron durante aproximadamente tres meses como parte de sus clases habituales de Comunicación Integral. Sus resultados fueron comparados con los de un grupo control. Se controló que antes de la intervención, el grupo control presentara resultados similares a los del grupo experimental en las variables estudiadas (Estudio 3).

Después de la recolección de datos, estos fueron analizados usando el Modelo de Ecuación Estructural para identificar el comportamiento de las variables y el análisis de varianza (ANOVA) para calcular los efectos de la intervención. También se calculó el Coeficiente Magnitud del Efecto de Cohen (Cohen, 1988) para determinar la fuerza estadística del efecto de intervención. Finalmente, se interpretaron los resultados de cada estudio por separado y finalmente, en conjunto.

\section{Resultados}

\section{Estudio 1}

Al aplicar el Modelo de Ecuación Estructural en el estudio 1 surgió el modelo que se presenta en la Figura 1, el cual constituyó la primera aproximación al comportamiento de las variables. Es importante notar que este modelo explicó el 34\% de la varianza en comprensión de lectura. Se muestra, además, que las variables que contribuyen con mayor fuerza a comprensión de lectura fueron decodificación y vocabulario. Las dimensiones de motivación fueron también predictores importantes de esta. Adicionalmente, de manera indirecta y directa, la variable socioeconómica presentó un efecto significativo sobre la misma. La variable clima alfabético del hogar mostró regresiones de un nivel alto en la mayoría de las variables intermedias y también de manera indirecta para comprensión de lectura. 


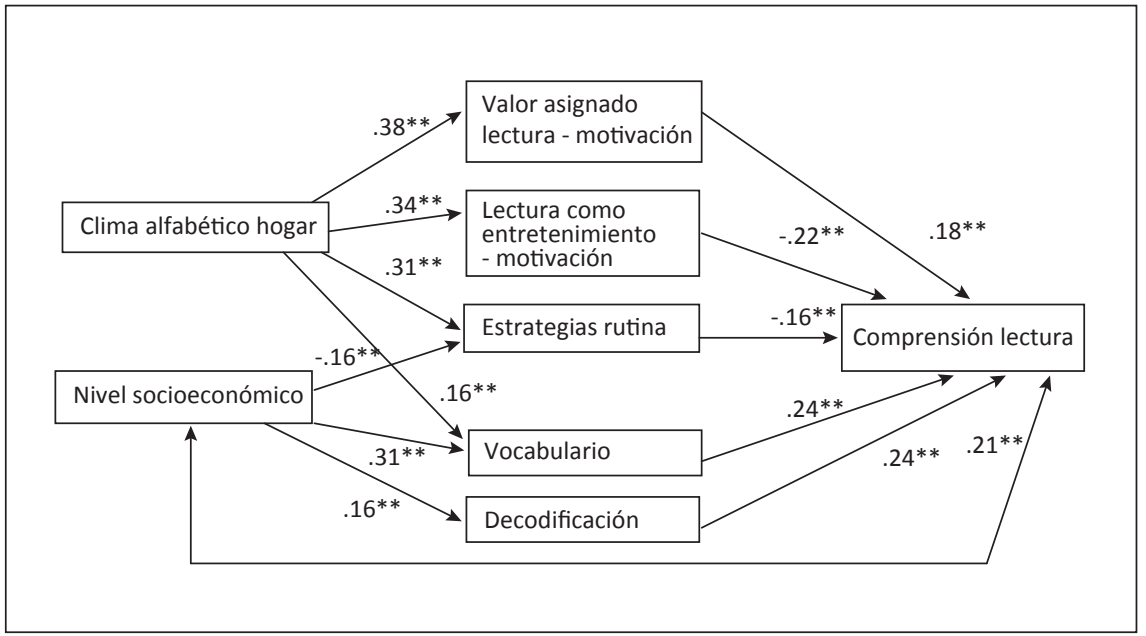

Figura 1. Modelo de Ecuación Estructural con contribuciones directas e indirectas de las variables predictoras sobre la comprensión de lectura distribuidas en dos niveles. El modelo explica el $34 \%$ de la varianza en comprensión de lectura.

\section{Estudio 2}

Después de dos mediciones del estudio 2, se obtuvo el modelo que se presenta en la Figura 2. El modelo explicó el 54,2\% de la varianza en comprensión de lectura en la segunda medición. Los resultados muestran relaciones de autorregresión fuertes. Esto comprueba la estabilidad de los instrumentos a lo largo del tiempo. Por ejemplo, la comprensión de lectura en el momento 2 (comprensión de lectura 2) se explicó de manera significativa por la misma variable en el momento 1.

También se evidencian relaciones longitudinales cruzadas (cross-lagged relationships), como en el caso de la variable valor asignado a la lecturamotivación sobre comprensión de lectura. Adicionalmente, se evidenciaron relaciones fuertes recíprocas entre decodificación y comprensión de lectura, y entre vocabulario y comprensión de lectura. Es decir, decodificación predijo comprensión de lectura, así como comprensión de lectura predijo decodificación. Y vocabulario predijo comprensión de lectura, así como comprensión de lectura predijo vocabulario. Tales relaciones recíprocas brindaron mayor poder de explicación al modelo obtenido.

Las variables de antecedentes mostraron una contribución importante en 114 I las variables intermediarias, y esas variables intermediarias en comprensión de 
lectura. Asimismo, se obtuvo que nivel socioeconómico constituyó un predictor significativo de comprensión de lectura.

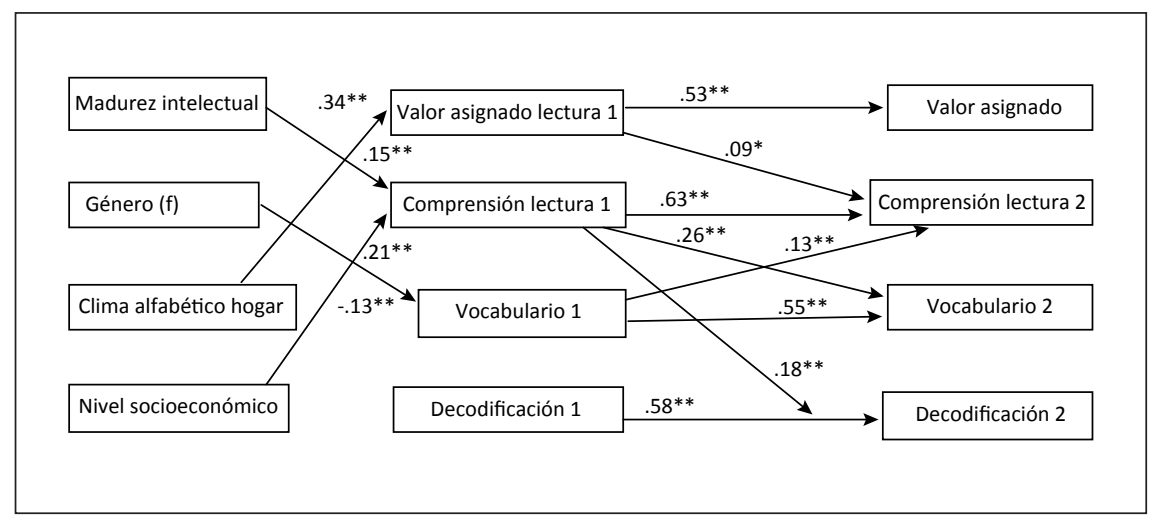

Figura 2. El modelo muestra la contribución de variables de antecedentes sobre el valor asignado a la lectura-motivación, comprensión de lectura, vocabulario y decodificación medidos en dos momentos. El modelo explica el $54,2 \%$ de la varianza en comprensión de lectura 2.

\section{Estudio 3}

El tercer estudio mostró el efecto del programa de intervención de lectura en la comprensión de lectura y la motivación hacia la lectura. Se utilizó el análisis de varianza para medidas repetidas.

Como se aprecia en la Figura 3, en relación con comprensión de lectura, el grupo experimental obtuvo puntajes más altos que el grupo control después de la intervención. Estos resultados fueron más fuertes en el grupo de nivel socioeconómico bajo.

En relación con las variables motivacionales, se esperaba que ambos grupos obtuvieran puntajes más bajos a lo largo del tiempo, como lo señala la literatura especializada (McKenna, Kear y Ellsworth, 1995; Wigfield y Guthrie, 1997); sin embargo, en relación con la variable interacción social-motivación, el grupo experimental obtuvo puntajes mayores, mientras que el grupo control mostró puntajes menores después de la intervención (Figura 4).

En relación con la variable valor asignado a la lectura-motivación, después de efectuada la intervención, el puntaje del grupo experimental decayó, pero en un grado mucho menor al del grupo control (Figura 5). 


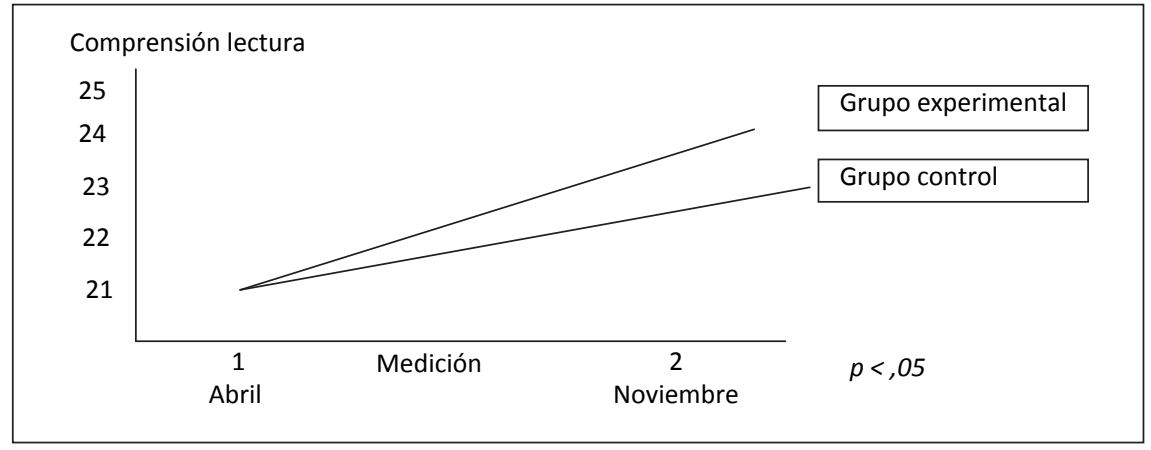

Figura 3. Medias de comprensión de lectura (prueba narrativa e informativa) en el pretest y el postest para cada grupo.

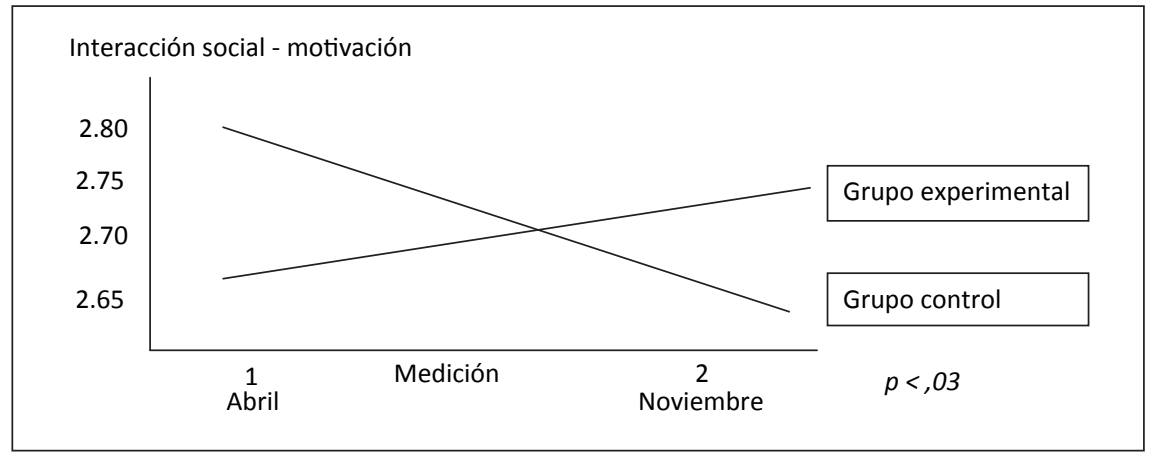

Figura 4. Medias de Interacción social-motivación en el pretest y el postest para cada grupo.

Valor asignado a lectura - motivación

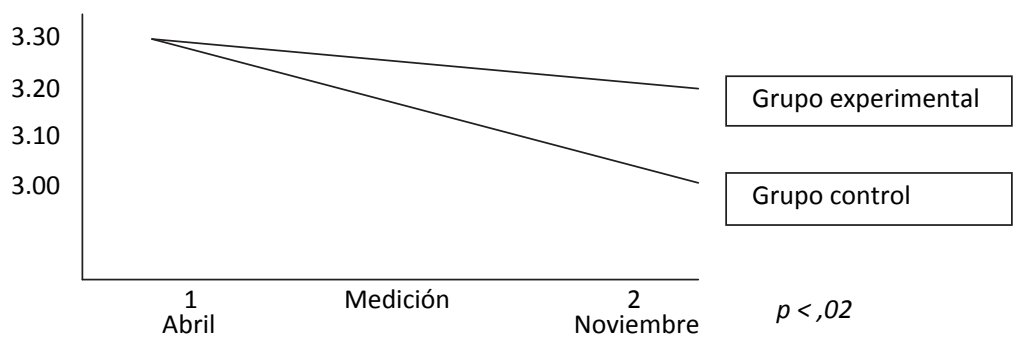

Figura 5. Medias de Valor asignado a la lectura-motivación en el pretest y el postest 116 I para cada grupo. 


\section{Discusión y Conclusiones}

Haciendo una recapitulación, las variables que contribuyen con mayor fuerza al desarrollo de la comprensión de lectura en estudiantes limeños son vocabulario, decodificación, motivación hacia la lectura y estatus socioeconómico. Se evidencia también un nivel de contribución fuerte del clima alfabético del hogar. En un nivel secundario de importancia se encuentran el género y la variable intelectual, que serían más importantes en los alumnos mayores (quinto grado) que en los menores (cuarto grado). Se puede resaltar también que las variables predictoras no actúan de manera aislada, más bien, se relacionan entre sí.

Estos resultados concuerdan con algunos estudios en Europa (Aarnoutse y van Leeuwe, 1998; Droop, 1999; van Elsäcker, 2002) y en Estados Unidos (Nagy, 1989; Perfetti, 1992; Sternberg, 1987), los cuales también evidencian la importancia de la decodificación y del vocabulario en el desarrollo de la comprensión de lectura; sin embargo, los resultados en Lima, añaden a lo anterior el importante rol del nivel socioeconómico y de la motivación, así como de la contribución de manera indirecta del clima alfabético del hogar.

En cuanto a las implicancias prácticas de este estudio, los maestros de Lima deberían considerar en el momento de planificar sus clases que la habilidad para decodificar continúa influyendo el desarrollo de la comprensión de lectura inclusive hasta el quinto grado de primaria; es decir, no afecta solo a los primeros grados de primaria, como muchos educadores aún creen.

Por otro lado, la consejería psicopedagógica a los padres de familia de Lima tendría que considerar crear conciencia de la importancia de la disponibilidad de materiales de lectura interesantes y del gran impacto de actividades relacionadas con la lectura en casa, enfatizando un ambiente social divertido que incluya actividades que refuercen los hábitos de lectura.

Asimismo, los resultados sustentan la importancia de la perspectiva de la motivación-compromiso (engagement perspective, Verhoeven y Snow, 2001) y de una aproximación de diversidad cultural al diseñar programas de intervención para el desarrollo de la comprensión de lectura. Adicionalmente, se resalta la importancia de introducir diferencias considerando la edad y el género - tomando en cuenta que las niñas suelen estar más motivadas - y el nivel intelectual en los estudiantes mayores.

Se debe tomar en cuenta que los resultados de esta investigación reflejan las características de un sector de la población de Lima; por lo tanto, no se pueden generalizar a toda la población del Perú. Asimismo, cabe resaltar que 
este estudio ha contribuido al desarrollo de la investigación en educación en el Perú al proponer un nuevo diseño de investigación, adaptar instrumentos a la población de Lima y explorar un grupo considerable de variables relacionadas con la comprensión de lectura. Así, se ha dado la posibilidad de comparar los resultados del Perú con aquellos de otros países. 


\section{Referencias}

Aarnoutse, C. y van Leeuwe, J. (1998). Relation between reading comprehension, vocabulary, reading pleasure, and reading frequency. Educational Research and Evaluation, 4(2) 143-166.

Anderson, J. (1993). Desde niñas: género y postergación en el Perú. Lima: UNICEF.

Apoyo, Opinión y Mercado. (2001). Informe gerencial de marketing. Lima: Apoyo, Opinión y Mercado.

Apoyo, Opinión y Mercado. (2003). Niveles socioeconómicos Perú. Lima: Apoyo, Opinión y Mercado.

Arakaki, M. (2004). Estrategias de lectura para el estudio y comprensión de textos en universitarios de primer año (tesis de licenciatura no publicada). Pontificia Universidad Católica del Perú, Lima.

Benavides, M. (2007). Lejos (aún) de la equidad: la persistencia de las desigualdades educativas en el Perú. En GRADE (Ed.), Investigación, políticas y desarrollo en el Perú (pp. 457-484). Lima: GRADE.

Brown, R.; Pressley, M.; van Meter, P. y Schuder, T. (1996). A quasi-experimental validation of Transactional Strategies Instruction with low-achieving second-graders. Journal of Educational Psychology, 20(1), 18-37.

Carreño, B. (2000). Comprensión de lectura al finalizar primaria en niños peruanos (tesis de maestría no publicada). Pontificia Universidad Católica del Perú, Lima.

Cohen, J. (1988). Statistical power analysis for the behavioral sciences. Hillsdale, NJ: Lawrence Erlbaum Associates.

Colegio de Profesores del Perú. (boletín web). Nuestra institución. Recuperado de http://www.cppe.org.pe/historia.php

Cueto, S.; Jacoby, E. y Pollitt, E. (1997a). Rendimiento en niños y niñas de zonas rurales y urbanas del Perú. Revista de Psicología de la PUCP, 14(1), 115-133.

Cueto, S.; Jacoby, E. y Pollitt, E. (1997b). Tiempo en la tarea y actividades educativas en escuelas rurales del Perú. Revista Latinoamericana de Estudios Educativos, 27(3), 105-120.

Droop, M. (1999). Effects of linguistic and cultural diversity on the development of reading comprehension (tesis doctoral). Universidad Radboud de Nimega, Holanda. Zeist: A-D Druck BV. 
Dunn, L. M.; Padilla, E. R.; Lugo, D. E. y Dunn, L. (1959). Test de vocabulario en imágenes Peabody. Adaptación hispanoamericana. Circle Pines, MN: AGS.

Gambrell, L. A.; Martin Palmer, B.; Codlin, R. M. y Anders Mazzoni, S. (1995). Assessing motivation to read. Maryland: NRRC.

Garner, R. (1998). Epilogue: Choosing to learn or not-learn in school. Educational Psychology Review, 10(2), 227-237.

Goodenough, F. (1969). Test de inteligencia infantil. Buenos Aires: Paidós.

Guthrie, J. T. y Alao, S. (1997). Designing contexts to increase motivations for reading. Educational Psychologist, 32(2), 95-105.

Guthrie, J. T. y Greaney, V. (1991). Literacy acts. En R. Barr, M. L. Kamil, P. Mosenthal y P. D. Pearson (Eds.), Handbook of reading research, Vol. 2. (pp. 68-96) Nueva York: Longman.

Guthrie, J. T.; van Meter, P.; McCann, A. D.; Wigfield, A.; Bennett, L.; Poundstone, C. C. et al. (1996). Growth of literacy engagement: Changes in motivations and strategies during con-cept-oriented reading instruction. Reading Research Quarterly, 31(3), 306-332.

Guthrie, J. T. y Wigfield, A. (2000). Engagement and motivation in reading. En M. L. Kamil, P. B. Mosenthal, P. D. Pearson y R. Barr (Eds.), Handbook of reading research, Vol. 3 (pp. 403-422). Mahwah, NJ: Erlbaum.

Heath, S. B. (1982). What no bedtime story means: Narrative skills at home and at school. Language in Society, 11, 49-76.

Heath, S. B. (1983). Ways with words: Language, life and work in communities and classrooms. Cambridge, MA: Harvard University Press.

International Association for the Evaluation of Educational Achievement. (2000). Framework and specifications for PIRLS assessment 2001. Chestnut Hill, MA: PIRLS International Study Center-Boston College.

McKenna, M. C.; Kear, D. J. y Ellsworth, D. A. (1995). Children's attitudes toward reading: A national survey. Reading Research Quarterly, 30, 934-956.

Martin, M. O.; Mullis, I. V. y Kennedy, A. M. (Eds.). (2003). PIRLS 2001. Technical report . Chestnut Hill, MA: Boston College.

Ministerio de Educación. (2000). Programa curricular de educación primaria de menores-ciclos 1, 2, 3. Lima: MED.

Ministerio de Educación. (2004). Lineamientos de política 2004-2006. La República (suplemento especial). 
Ministerio de Educación. (2007). Plan estratégico institucional 2007-2011. Documento de trabajo. Recuperado de www.minedu.gob.pe/ Publicaciones/PEIMED-05.XI.07.pdf.

Morales, S. (1999). Comprensión de lectura en alumnos de cuarto y quinto grados de primaria de Lima. Revista de Psicología de la PUCP, 17(2), 263-282.

Nagy, W. E. (1989). Teaching vocabulary to improve reading comprehension (NCTE report № 52384). Urbana, IL: NCTE.

OECD y UNESCO. (2003). Literacy skills for the world of tomorrow. Further results from PISA 2000. Recuperado de http://www.pisa. oecd.org/ dataoecd/43/9/33690591.pdf.

Perfetti, C. A. (1992). The representation of problem in reading acquisition. En P. B. Gough, L. C. Ehri y R. Treitman (Eds.), Reading acquisition (pp. 145174). Hillsdale, NJ: Erlbaum.

Ramírez, E. (1997). Expresar, comunicar, comprender. Lima: Línea y Punto.

Serpell, R. (2001). Cultural dimensions of literacy promotion and schooling. En L. Verhoeven y C. E. Snow (Eds.), Literacy and motivation (pp. 243-274). Mahwah, NJ: Erlbaum-IEA.

Sternberg, R. J. (1987). Most vocabulary is learned from context. En M. G. Mckeown y M. E. Curtis (Eds.), The nature of vocabulary acquisition (pp. 89-105). Hillsdale, NJ: Erlbaum.

Street, B. (2001). Literacy empowerment in developing societies. En L. Verhoeven y C. E. Snow (Eds.), Literacy and motivation (pp. 291-300). Mahwah, NJ: Erlbaum-IEA.

Thorne, C. (1991). Beginning reading in Lima (tesis doctoral). Universidad Radboud de Nimega. Nimega: Quick Print.

Unidad de Medición de la Calidad. (boletín web). Laboratorio Latinoamericano de Evaluación de la Calidad Escolar. Recuperado de: http://www2.minedu. gob.pe/umc/ index 2.php?v_codigo $=76 \&$ v_plantilla $=2$

van der Westen, M. (1994). Literacy education and gender: The case of Honduras. En L. Verhoeven (Ed.), Functional literacy (pp. 257-278). Amsterdam: Benjamins.

van Elsäcker, W. (2002). Development of reading comprehension: the engagement perspective (tesis doctoral). Universidad de Nimega, Holanda. Entschede: Feboprint. 
Verhoeven, L. y Snow, C. E. (2001). Literacy and motivation. Mahwah, NJ: Erlbaum-IEA.

Walberg, H. J. y Wang, M. C. (1989). Effective educational practices and provisions for individual differences. En M. C. Wang, M. C. Reynolds y H. J. Walberg (Eds.), Handbook of special education: Research and practice, Vol. 1 (pp. 113-128). Nueva York: Pergamon.

Wigfield, A. (1997). Reading motivation: A domain-specific approach to motivation. Educational Psychologist, 32(2), 59-68.

Wigfield, A. y Guthrie, J. T. (1997). Relations of children's motivation for reading to the amount and breadth of their reading. Journal of Educational Psychology, 89(3), 420-432.

Wigfield, A.; Guthrie, J. y Mc Gough, K. (1996). A questionnaire measure of children`s motivation for reading. Maryland: NRRC.

Zavala, V. (2001). Oralidad y escritura en la educación bilingüe (a propósito de la interculturalidad). Lima: MED-GTZ-KFW. 


\section{Anexo}

\section{Cuestionario Estrategias de Lectura}

\section{RSQ}

\section{Protocolo}

Código:

Nombre:

Grado: Sección:

Colegio:

Fecha:

Profesor(a): Pt

\section{Instrucciones}

En el colegio, a veces tienes que leer algunos textos como los cuentos o textos informativos, como en sociales. Trata de recordar la manera como sueles leerlos.

Vas a responder algunas preguntas relacionadas a lo que sueles hacer antes, durante y después de leer un texto. Esto no es un examen. Solamente queremos saber más sobre tu manera de leer. Toda respuesta estará bien si es verdadera. Cada persona puede tener una respuesta diferente. Te pedimos que seas honesto al responder las preguntas.

Debes indicar con qué frecuencia (cuántas veces) realizas algunas cosas: siempre, a veces o (casi) nunca.

- $\quad$ Siempre significa que las realizas cada vez o la mayoría de las veces.

- A veces significa que no las haces todas las veces, pero sí de manera ocasional.

- (Casi) Nunca significa que no las sueles hacer o que las has realizado sólo una vez

Cuando hayas elegido tu respuesta, la marcas con un aspa o una cruz. A continuación te presentamos unos ejemplos.

Ejemplos

\begin{tabular}{llll}
\hline E.1 Yo voy al colegio & Siempre & A veces & (Casi) Nunca \\
\hline E.2 Yo voy al teatro & Siempre & A veces & (Casi) Nunca \\
\hline E.3 Yo visito a mis primos & Siempre & A veces & (Casi) Nunca \\
\hline
\end{tabular}


Si deseas cambiar alguna opción que ya marcaste, la tachas y vuelves a marcar con un aspa la respuesta correcta. Si tienes alguna duda, levanta la mano y pregunta.

¿Estás listo(a)? Ahora empezaremos juntos con las preguntas.

\section{Antes de leer}

Cuando tienes que leer un cuento o un texto informativo en el colegio ¿qué haces antes de leerlo? Piensa cuidadosamente y sé lo más honesto(a) posible al responder las siguientes preguntas.

1. Antes de leer, reviso el texto para ver si me va a gustar.

Siempre A veces (Casi) Nunca

2. Antes de leer, miro los dibujos o figuras para ver de qué se trata el texto.

Siempre A veces (Casi) Nunca

3. Antes de leer, miro el texto para ver si es difícil.

Siempre A veces (Casi) Nunca

4. Miro los títulos y subtítulos antes de leer.

Siempre A veces (Casi) Nunca

5. Antes de leer, doy una revisada rápida a todo el texto.

Siempre A veces (Casi) Nunca

6. Miro las palabras impresas en negrita antes de empezar a leer.

Siempre A veces (Casi) Nunca

7. Antes de leer, trato de imaginar de qué se trata el texto.

Siempre A veces (Casi) Nunca

8. Me hago algunas preguntas sobre el texto antes de leer. 


\title{
Durante la lectura
}

¿Qué sueles hacer mientras lees un texto narrativo o informativo?

Piensa cuidadosamente y sé lo más honesto(a) posible al responder.

1. Durante la lectura, trato de recordar lo que leo.

Siempre A veces (Casi) Nunca

2. Cuando no comprendo alguna palabra, busco alguna otra parte en el texto que sí entienda.

Siempre A veces (Casi) Nunca

\author{
and
}

3. Si hay algo que no entiendo, retrocedo en el texto.

Siempre A veces (Casi) Nunca
4. Mientras leo, verifico si el texto era como yo me lo había Siempre A veces (Casi) Nunca imaginado antes de leerlo.

5. Si mientras leo no sé alguna palabra, la apunto.

Siempre A veces (Casi) Nunca

6. Si no sé alguna palabra, la busco en el diccionario.

Siempre A veces (Casi) Nunca

7. Hago apuntes mientras leo.

Siempre A veces

(Casi) Nunca

8. Si no entiendo alguna palabra, trato de buscar el significado leyendo las oraciones que la Siempre A veces (Casi) Nunca rodean.

9. Si releo una oración y sigo sin entenderla, continúo leyendo para buscar más pistas que me Siempre A veces (Casi) Nunca ayuden. 


\section{Después de la lectura}

¿Qué sueles hacer después de leer un texto narrativo o informativo?

Piensa cuidadosamente y sé lo más honesto(a) posible al responder las siguientes preguntas.

\begin{tabular}{lccc}
\hline $\begin{array}{l}\text { 1. Hago un resumen después de } \\
\text { leer }\end{array}$ & Siempre & A veces & (Casi) Nunca \\
\hline $\begin{array}{l}\text { 2. Escribo las ideas más } \\
\text { importantes después de leer. }\end{array}$ & Siempre & A veces & (Casi) Nunca \\
\hline $\begin{array}{l}\text { 3. Hago un esquema o dibujo } \\
\text { después de leer. }\end{array}$ & Siempre & A veces & (Casi) Nunca \\
\hline $\begin{array}{l}\text { 4. Después de leer, reviso el texto } \\
\text { por segunda vez. }\end{array}$ & Siempre & A veces & (Casi) Nunca \\
\hline $\begin{array}{l}\text { 5. Leo el texto dos veces: rápido la } \\
\text { primera vez y lento la segunda } \\
\text { vez. }\end{array}$ & Siempre & A veces & (Casi) Nunca \\
\hline $\begin{array}{l}\text { 6. Después de leer, me pregunto: } \\
\text { ¿entendí todo? }\end{array}$ & Siempre & A veces & (Casi) Nunca \\
\hline $\begin{array}{l}\text { 7. Después de leer, me pregunto: } \\
\text { ¿Cuál es la idea principal de este } \\
\text { texto? }\end{array}$ & Siempre & A veces & (Casi) Nunca \\
\hline $\begin{array}{l}\text { 8. Después de leer, trato de pensar } \\
\text { ahora? } \\
\text { en las ideas más importantes. }\end{array}$ & Siempre & A veces & (Casi) Nunca \\
\hline $\begin{array}{l}\text { 9. Después de leer, leo algunas } \\
\text { partes de nuevo. }\end{array}$ & Siempre & A veces & (Casi) Nunca \\
\hline $\begin{array}{l}\text { 11. Después de leer, me pregunto: } \\
\text { (C) }\end{array}$ & Siempre & A veces & (Casi) Nunca \\
\hline
\end{tabular}




\section{Cuestionario Clima Alfabético del Hogar}

\section{Clima Alfabético en el Hogar}

(Adaptado de Van Elsäcker, 2001)

1. ¿Con qué frecuencia lees en casa?

2. ¿Con qué frecuencia lees el periódico?

3. ¿Estás suscrito a alguna revista infantil?

4. ¿Cuántos libros en casa son tuyos?

5. ¿Cuántos libros tienen tus padres en casa? ¿Cuántos libros hay en tu casa? (aprox.)

6. ¿Con qué frecuencia te leían tus padres cuando eras pequeño(a)?

7. ¿Con qué frecuencia te leen ahora tus padres?

8. ¿Cómo reaccionan tus padres cuando lees?

9. Cuando empiezas con un libro clo lees hasta el final?

10. Cuando lees en casa ¿cuánto tiempo lo haces?

11. ¿Cuentas con algún lugar en casa donde puedas leer con tranquilidad?

12. Cuando estás leyendo un libro ¿cuántas veces te preguntan tus padres sobre lo que se trata?

13. ¿Con qué frecuencia cuentas en casa sobre un libro que has leído?

14. ¿Con qué frecuencia buscas en casa una palabra en el diccionario?

15. ¿Con qué frecuencia lees en voz alta a alguien en casa?

16. ¿Con qué frecuencia tú o alguien de tu familia cuenta una historia graciosa o interesante sin estar leyendo un libro?

17. ¿Con qué frecuencia escribes una historia o cuento en casa?

18. ¿Con qué frecuencia cantan canciones juntos en casa?

19. ¿Con qué frecuencia vas a la biblioteca de tu escuela, distrito o de la ciudad? 


\section{Clima Alfabético en el Hogar}

Hoja de respuesta

Código:

Nombre:

Grado: Sección:

Colegio:

Fecha:

Profesor(a): $\mathrm{Pt}$

\section{Instrucciones}

A continuación vas a responder algunas preguntas relacionadas a la actividad de leer. Para esto tienes que elegir una opción entre todas las opciones de respuesta.

Esto no es un examen. Solamente queremos saber más sobre ti y tu manera de leer. No existe una única respuesta correcta. Cada persona puede tener una respuesta diferente. Solo tienes que ser lo más sincero posible al responder. A continuación te presentamos algunos ejemplos.

Ejemplos

E.1. ¿Con qué frecuencia visitas a tus primos y tíos?

\begin{tabular}{|l|l|l|l|}
\hline ( ) Nunca o casi nunca & ( ) 1 vez/ 4 semanas & ( ) 1 vez/ 2 ó $3 \mathrm{sem}$. & ( ) 1 vez / $1 \mathrm{sem}$ \\
\hline
\end{tabular}

E.2. ¿Con qué frecuencia vas a la playa en verano?

\begin{tabular}{|l|l|l|l|} 
() Nunca o casi nunca & ( ) $1 \mathrm{vez} / 4$ semanas & () $1 \mathrm{vez} / 2$ ó $3 \mathrm{sem}$ & () $1 \mathrm{vez} / \mathrm{sem}$ \\
\hline
\end{tabular}

E.3 ¿Cuántos primos tienes?

\begin{tabular}{|l|l|l|}
\hline () uno o dos & () Entre 3 y 6 & () Más de 7 \\
\hline
\end{tabular}

Si deseas cambiar alguna opción ya marcada, la tachas y vuelves a marcar con una cruz la respuesta correcta. Si tienes alguna duda, levanta la mano y pregunta.

128 I ¿Estás listo(a)? Ahora trabajarás en silencio con las preguntas. 


\section{Clima Alfabético en el Hogar}

\begin{tabular}{|c|c|c|c|c|c|c|c|c|c|}
\hline 1 & $\begin{array}{l}\text { () Nunca o } \\
\text { casi nunca }\end{array}$ & \multicolumn{2}{|c|}{$\begin{array}{l}\text { () } 1 \text { vez/ } 4 \\
\text { semanas }\end{array}$} & \multicolumn{2}{|c|}{$\begin{array}{l}\text { () } 1 \text { vez/ } 2 \text { ó } \\
3 \text { semanas }\end{array}$} & $\begin{array}{l}\text { ( ) } 1 \mathrm{vez} / \\
\text { sem. }\end{array}$ & \multicolumn{2}{|c|}{$\begin{array}{c}\text { ( ) } 2 \text { ó } 3 \text { veces/ } \\
1 \text { sem }\end{array}$} & $\begin{array}{c}\text { ( ) } 4 \text { o más veces/ } \\
1 \text { sem. }\end{array}$ \\
\hline 2 & \multicolumn{2}{|c|}{$\begin{array}{c}\text { ( ) Nunca o casi } \\
\text { nunca }\end{array}$} & \multicolumn{2}{|c|}{$\begin{array}{l}\text { () } 1 \text { vez/ } 4 \\
\text { semanas }\end{array}$} & $\begin{array}{l}\text { () } 1 \text { vez/2 ó } \\
3 \text { semanas }\end{array}$ & \multicolumn{2}{|c|}{$\begin{array}{l}\text { () } 1 \mathrm{vez} / 1 \\
\text { sem. }\end{array}$} & $\begin{array}{c}\text { ( ) } 2 \text { ó } 3 \text { veces } \\
1 \text { sem }\end{array}$ & $\begin{array}{l}\text { () } 4 \text { o más } \\
\text { veces } / 1 \text { sem. }\end{array}$ \\
\hline 3 & ( ) Sí & & & & & () No & & & \\
\hline
\end{tabular}

\begin{tabular}{|l|l|l|l|l|}
\hline 4 & ( ) 1 o ninguno & ( ) Entre 2 y 4 & ( ) Entre 5 y 10 & () Más de 10 \\
\hline
\end{tabular}

\begin{tabular}{|l|l|l|l|l|l|}
\hline 5 & ( ) Más de 200 & ( ) Entre 100 y 200 & ( ) Entre 50 y 100 & ( ) Entre 10 y 50 & ( ) Menos de 10 \\
\hline
\end{tabular}

\begin{tabular}{|l|c|c|c|c|c|c|c|}
\hline 6 & $\begin{array}{c}\text { ( ) Nunca o } \\
\text { casi nunca }\end{array}$ & $\begin{array}{c}\text { () } 1 \text { vez/ } 4 \\
\text { semanas }\end{array}$ & $\begin{array}{c}\text { ( ) } 1 \text { vez/ } 2 \text { ó } 3 \\
\text { semanas }\end{array}$ & $\begin{array}{c}\text { ( ) } 1 \text { vez/ } 1 \\
\text { sem }\end{array}$ & $\begin{array}{c}\text { ( ) } 2 \text { ó } 3 \text { veces/ } \\
1 \text { sem }\end{array}$ & $\begin{array}{c}\text { ( ) } 4 \text { o más veces/ } \\
1 \text { sem. }\end{array}$ \\
\hline 7 & $\begin{array}{c}\text { () Nunca o casi } \\
\text { nunca }\end{array}$ & $\begin{array}{c}\text { () } 1 \text { vez/ } 4 \\
\text { semanas }\end{array}$ & $\begin{array}{c}\text { () } 1 \text { vez/ } 2 \\
\text { semanas }\end{array}$ & $\begin{array}{c}\text { () } 1 \text { vez/ } 1 \\
\text { sem }\end{array}$ & $\begin{array}{c}\text { ( ) } 2 \text { ó } 3 \text { veces/ } \\
1 \text { sem }\end{array}$ & $\begin{array}{c}\text { ( ) } 4 \text { o más } \\
\text { veces/ } 1 \text { sem. }\end{array}$ \\
\hline
\end{tabular}

\begin{tabular}{|l|l|l|l|l|}
\hline 8 & ( ) Ni se enteran & $\begin{array}{c}\text { ( ) Parecen satisfechos, aunque no } \\
\text { me dicen nada }\end{array}$ & $\begin{array}{c}\text { ( ) A veces me } \\
\text { alientan }\end{array}$ & $\begin{array}{c}\text { ( ) Siempre me } \\
\text { alientan }\end{array}$ \\
\hline 9 & () Nunca & ( ) A veces & () Casi siempre \\
\hline 10 & ( ) Más de 1 hora & $\begin{array}{c}\text { ( ) Entre } 30 \text { min. y } 1 \\
\text { hora }\end{array}$ & $\begin{array}{c}\text { () Entre } 15 \text { min. y } \\
30 \text { min. }\end{array}$ & ( ) Máximo 15 min. \\
\hline
\end{tabular}

\begin{tabular}{|c|c|c|c|}
\hline 11 & () No & () A veces & ( ) Sí \\
\hline 12 & () Nunca & () A veces & () Frecuentemente \\
\hline
\end{tabular}

\begin{tabular}{|c|c|c|c|}
\hline 13 & () Nunca & () A veces & () Frecuentemente \\
\hline 14 & () Nunca & () A veces & () Frecuentemente \\
\hline
\end{tabular}

\begin{tabular}{|c|c|c|c|}
\hline 15 & () Nunca & () A veces & () Frecuentemente \\
\hline 16 & () Nunca & () A veces & () Frecuentemente \\
\hline
\end{tabular}

\begin{tabular}{|c|c|c|c|c|c|}
\hline 17 & $\begin{array}{c}\text { ( ) } 1 \text { o más } \\
\text { veces/ } 1 \text { sem. }\end{array}$ & $\begin{array}{c}\text { ( ) } 1 \text { vez/ } 2 \text { ó } 3 \\
\text { sem. }\end{array}$ & $\begin{array}{c}\text { ( ) } 1 \text { vez/ } 4 \\
\text { semanas }\end{array}$ & $\begin{array}{c}\text { ( ) } 1 \text { vez/ } 2 \text { ó } 3 \\
\text { meses }\end{array}$ & $\begin{array}{c}\text { ( ) Nunca o casi } \\
\text { nunca }\end{array}$ \\
\hline
\end{tabular}

\begin{tabular}{|c|c|c|c|c|c|c|}
\hline 18 & $\begin{array}{c}\text { () Nunca o } \\
\text { casi nunca }\end{array}$ & $\begin{array}{c}\text { ( ) } 1 \text { vez/ } 4 \\
\text { semanas }\end{array}$ & $\begin{array}{c}\text { ( ) } 1 \text { vez/ } 2 \\
\text { semanas }\end{array}$ & $\begin{array}{c}\text { ( ) } 1 \text { vez/ } 1 \\
\text { sem. }\end{array}$ & $\begin{array}{c}\text { ( ) } 2 \text { ó } 3 \text { veces/ } \\
1 \text { sem. }\end{array}$ & $\begin{array}{c}\text { ( ) } 4 \text { o más } \\
\text { veces } / 1 \text { sem. }\end{array}$ \\
\hline
\end{tabular}

\begin{tabular}{|c|c|c|c|c|c|}
\hline 19 & $\begin{array}{c}\text { () } 1 \text { o más } \\
\text { veces/ } 1 \text { sem. }\end{array}$ & $\begin{array}{c}\text { ( ) } 1 \text { vez/ } 2 \\
\text { sem. }\end{array}$ & $\begin{array}{c}\text { ( ) } 1 \text { vez/ } 4 \\
\text { semanas }\end{array}$ & $\begin{array}{c}\text { ( ) } 1 \text { vez/ } 2 \text { ó } 3 \\
\text { meses }\end{array}$ & $\begin{array}{c}\text { ( ) Nunca o casi } \\
\text { nunca }\end{array}$ \\
\hline
\end{tabular}

Manuscrito recibido: 30 de julio, 2011

Revisado: 25 de octubre, 2011 Aceptado: 06 de noviembre, 2011 